\section{PTH-040 WHAT IS THE UTILITY OF CAPSULE ENDOSCOPY IN PATIENTS WITH OBSCURE GASTROINTESTINAL BLEEDING AND CHRONIC RENAL FAILURE?}

doi:10.1136/gutjnl-2013-304907.527

1."P S Sidhu, 'M E McAlindon, 'R Sidhu. 'Gastroenterology, Royal Hallamshire Hospital, Sheffield Teaching Hospitals, Sheffield, UK

Introduction Anaemia is a common complication of chronic renal failure (CRF). The cause of the anaemia is often multifactorial including erythropoietin deficiency, uraemic platelet dysfunction, absolute iron deficiency anaemia (IDA) and overt bleeding (OB). Capsule endoscopy (CE) has developed an important role in the investigative pathway of obscure gastrointestinal bleeding (OGB). We conducted a study to evaluate the utility of CE in such patients after negative bidirectional endoscopies and their subsequent management consequences.

Methods We retrospectively reviewed our data set and isolated patients with OGB and CRF. This data set was scrutinised to elucidate demographics, factors that increase yield and their subsequent management.

Results Of the 1324 patients investigated, 4.1\%(n = 54) had CRF. The mean age was 68 years (range $=37-86$ ) and males consisted of $56 \%$ of the new cohort $(n=33)$. The indications for CE was IDA in $61 \%(\mathrm{n}=33)$ of patients and $\mathrm{OB}$ in the remaining $39 \%$. The majority $89 \%(n=48)$ of patients had significant other co-morbidity which included cardiovascular (13), diabetes (10), haematological (8), cancers (7), respiratory disease (7) and chronic liver disease (3). 3 patients were on warfarin and 4 patients were transfusion dependent. The diagnostic yield (as defined by lesions responsible for OGB) identified on CE was $48 \%(\mathrm{n}=26)$. Angioectasia was the commonest diagnosis identified in $33 \%$ of patients $(n=18)$. Other findings were ulcers and erosions $22 \%(n=12)$, fresh blood without a definite bleeding site $17 \%(n=9)$, strictures in $7 \%(n=4)$, whilst crohns and polyps were seen in $2 \%(n=1)$ each respectively. CE identified significant lesions within the upper gastrointestinal tract in $19 \%(n=10)$ of patients. There was no significant difference in the diagnostic yield between those with IDA and OB $(p=0.53)$ and between the sexes $(p=0.76)$. Management was altered in $44 \%(n=24)$ of those with a positive yield, in the form of further procedures $(48 \%$, $\mathrm{n}=12)$. These procedures included push-enteroscopies $58 \%(n=7)$, double balloon enteroscopies $25 \%(n=3)$ and 3 OGDs. In the same group, argon photocoagulation therapy was applied to $83 \%(n=10)$ of patients.

Conclusion CE has a high diagnostic yield in patients with CRF with a positive outcome on subsequent management. Small bowel angioectasia is the commonest finding. CE should be considered in the work up of patients with CRF and OGB.

Disclosure of Interest None Declared.

\section{PTH-041 POLYP CANCERS: WHEN IS SURGICAL RESECTION NEEDED?}

doi:10.1136/gutjnl-2013-304907.528

1,"R Rameshshanker, 'A King, 'S Catnach, 'S Cerys, 'B Macfarlane. 'Gastroenterology, West Herts NHS Trust, Hemel Hempstead Hospital, UK

Introduction The Bowel Cancer Screening Program (BCSP) has been successful in detecting early stage bowel cancer, including polyp cancers. Most polyp cancers are not recognised as such at the index colonoscopy and are managed initially with endoscopic resection. There are histological criteria to predict high risk polyp cancers and need for further surgical resection. The decision is not always clear cut, and who to refer for surgery is difficult. A single centre experience is reported
Methods Polyp cancers detected through the BCSP at West Herts NHS Trust between August 2008 and December 2011 were identified. Endoscopy reports, polyp histology, surgical referrals and surgical histology were analysed.

Results 1334 colonoscopies were performed and 25 polyp cancers which had been managed initially with endoscopic resection were identified. Twenty four were within recto-sigmoid area, size range 1-4 cm. No polyp cancers were poorly differentiated. Patient age range was 60-74 yrs, median 66 yrs.

Fourteen patients had endoscopic resection (ER) alone: thirteen remain disease free at 1 year endoscopic follow up, one patient did not attend. Of the pedunculated polyp cancers, two were Haggitt $1(\mathrm{H} 1)$, four Haggitt $2\left(\mathrm{H}_{2}\right)$ and three Haggitt 3 (H3). The resection margin (RM) ranged from 1.5 to $12 \mathrm{~mm}$. There was tumour budding in just one, but the RM was $12 \mathrm{~mm}$. No case had lympho vascular invasion (LVI). Of the sessile polyp cancers there were two Kikuchi 1 (K1), two Kikuchi 2 (K2) and one Kikuchi 3(K3). There was budding in one case, a K1 polyp, and one LVI in a K2 polyp but with $4 \mathrm{~mm}$ RM. The K3 polyp had budding, no LVI and RM 0.5-MRI and CT scans were negative in this patient.

Eleven patients were referred for surgery post ER. Nine patients had no residual tumour. In these nine patients, polyp features dictating referral were: one $\mathrm{H}_{2}$ with budding and $0.5 \mathrm{~mm} \mathrm{RM}$, one $\mathrm{H} 3$ with LVI and $1 \mathrm{~mm} \mathrm{RM}$, one $\mathrm{H3}$ with budding and $1 \mathrm{~mm} \mathrm{RM}$, one $\mathrm{H} 3$ with $0.5 \mathrm{~mm} \mathrm{RM}$, one H3 RM not clear, one H3 with $2 \mathrm{~mm}$ RM no LVI or budding, one K2 with budding LVI and $0.5 \mathrm{~mm} \mathrm{RM}$, two K3 RM not clear. Two patients had cancer: one pT1pN1Mx (polyp cancer: H3 no budding or LVI RM 1mm) and pT1pN0Mx (polyp cancer: K2 with budding RM 0.5)

Conclusion When all three high risk histological features (budding, LVI, RM $<1 \mathrm{~mm}$ ) are absent or present a decision re surgical referral can be straightforward. Using these parameters, in our study, probably one patient more should have been referred for surgical resection, and one patient less. The decision challenge lies when just one or two parameters are present. A pessimistic view is that $82 \%$ of our patients had unnecessary surgery, an optimistic one that $18 \%$ had appropriate surgery. Age and associated morbidity are also important factors illustrating the value of the MDT in these challenging decisions

Disclosure of Interest None Declared.

\section{PTH-042 IRON DEFICIENCY ANAEMIA IN YOUNG MALES: DO GI SYMPTOMS AND HAEMOGLOBIN LEVEL AFFECT DIAGNOSTIC YIELD?}

doi:10.1136/gutjnl-2013-304907.529

1."R Kalla, 'S Campbell, 'R Willert. 'Gastroenterology, Manchester Royal Infirmary, Manchester, UK

Introduction Young males with iron deficiency (IDA) routinely undergo endoscopy. There is paucity of data on the diagnostic yield of bi-directional endoscopy, level of $\mathrm{HB}$ that requires investigation and the yield in symptomatic vs. asymptomatic young men.

We aim to study the diagnostic yield of bi-directional endoscopy in young males and the influence of gastrointestinal symptoms and HB level on yield.

Methods Data was collected from UNISOFT endoscopy software for all male patients referred for iron deficiency anaemia. Strict inclusion criteria were set and defined as: 1 . Age $20-50$ years 2 . No prior gastrointestinal (GI) diagnosis 3. Blood indices - hypoferritinemia confirming IDA at the time of referral

A total of 36 patients were identified from January 2010 to July 2012. A retrospective review of blood parameters, symptoms, nonsteroidal anti-inflammatory drug (NSAID) use, endoscopic findings 
and histologies were recorded. Data was recorded on an excel sheet and analysed using SPSS version 15 .

Results A total of 36 patients were included in the study. The median age was 42 years. Eleven percent $(n=4)$ were referred from general medicine, $22 \%(\mathrm{n}=8)$ from general surgery and $67 \%$ $(n=24)$ from Gastroenterology for endoscopic investigations. Median HB level was $10 \mathrm{~g} / \mathrm{dL}$. There were 4 patients on NSAIDs prior to referral.

Gastroscopy was performed in all patients and was normal in $94 \%(\mathrm{n}=34)$. The remaining $6 \%(\mathrm{n}=2)$ were diagnosed with $\mathrm{h}$ pylori gastritis $(n=1)$, and benign pyloric stenosis $(n=1)$. Duodenal (D2) biopsies were performed in 32 patients (89\%), of which 1 patient was diagnosed with coeliac disease on histology. Three of the 4 patients with no D2 biopsies had a normal coeliac blood screen. Lower GI endoscopy (colonoscopy $n=30$, flexible sigmoidoscopy $n=3)$ was performed in $33(92 \%)$, of which $85 \%(n=28 / 33)$ were macroscopically normal. Five patients had pathology encountered at endoscopy, these included adenomas $(n=3)$, ulcerative colitis $(n=1)$ and caecal carcinoma $(n=1)$.

Patients with IDA and no GI symptoms ( $\mathrm{n}=21$ ); endoscopic diagnostic yield was $5 \%(n=1)$ for gastroscopy and $11 \%(n=2 / 19)$ for colonoscopy. The diagnostic yield in symptomatic patients was $7 \%(n=1)$ for gastroscopy and $21 \%(n=3 / 14)$ for colonoscopy.

There were 11 patients with IDA and an HB level over $11 \mathrm{~g} / \mathrm{dL}$. All patients underwent gastroscopy with D2 biopsies and 10 patients underwent colonoscopy. All endoscopic investigations including $\mathrm{D} 2$ biopsies were normal.

Conclusion IDA in young males should always be investigated with bi-directional endoscopy, irrespective of symptoms. Although a small cohort, our study shows that the diagnostic yield is higher amongst patients with IDA and GI symptoms as well as IDA with an HB level below 11g/dL.

Disclosure of Interest None Declared.

\section{PTH-043 DIFFICULT BILIARY ACCESS: EARLY EXPERIENCE WITH THE NEW DOUBLE WIRE TECHNIQUE COMPARED TO CONVENTIONAL PRECUT AT ERCP}

doi:10.1136/gutjnl-2013-304907.530

1."R Rameshshanker, 'B Macfarlane, 'A King, 'A Leahy. 'Gastroenterology, Watford General Hospital, Watford, UK

Introduction Selective cannulation of the common bile duct (CBD) during endoscopic retrograde cholangiopancreatography (ERCP) can be technically challenging. The precut technique is a tried and tested method of gaining biliary access when standard cannulation with either contrast or guidewire has failed. However, it does have a recognised higher risk of causing pancreatitis and when used it is currently advised to place a prophylactic pancreatic stent. A new technique, termed double guidewire, involves leaving a wire in the pancreatic duct to provide a "roadmap" for subsequent biliary wire cannulation.

Objectives To report our initial experience for the success and complication rate of the double guidewire technique.

Methods Retrospective analysis of all patients who underwent ERCP between April 2010 to April 2012 at our institution. Observed differences were tested with the Fisher's exact test.

Results 484 ERCP procedures, all having initial cannulation with either a single guidewire or contrast. Overall successful biliary cannulation for first ever ERCP was achieved in 95\%. Attempted CBD cannulation was facilitated by double guidewire in 20 patients and precut in 19 patients. CBD cannulation was successful in 19/20 (95\%) double guidewire patients and 15/19 (79\%) precut patients $(\mathrm{p}=\mathrm{NS})$.
Post-ERCP pancreatitis occurred in all procedures were $11 / 484$ $(2.2 \%)$, double guidewire $3 / 20(15 \%)(p<0.02)$, precut $1 / 19(5.3 \%)$. Prophylactic pancreatic stents were placed in 2 double guidewire patients and no precut patients. In the double guidewire group, patients with pancreatitis stayed longer (mean 8 days) in the hospital when compared to precut group (mean 3 days).

Conclusion Deep CBD cannulation could be equally achieved with the help of either the double guidewire or the precut technique. There was a significant increased risk of pancreatitis with the double guidewire technique presumably related to inadvertent and repeated wire cannulations of the pancreatic duct. We find that the double guidewire technique is technically easy and currently use it if pancreatic duct cannulation occurs on $>3$ occasions. However, when used we now also place a prophylactic pancreatic stent at the end of the procedure.

Conflicts of interest none

Disclosure of Interest None Declared.

\section{PTH-044 THE SAFETY OF ERCP IN PATIENTS AGED 90 AND ABOVE}

doi:10.1136/gutjnl-2013-304907.531

1,"R Pandey, 'M Khan, ${ }^{1} \mathrm{~N}$ Van Someren, ${ }^{1} \mathrm{~K}$ Besherdas. 'Gastroenterology, Barnet \& Chase Farm NHS Trust, London, UK

Introduction The ageing population in the UK means that biliary diseases are becoming more common. Endoscopic Retrograde Cholangiopancreatography (ERCP) is therefore increasingly being used as a therapeutic strategy. However, the elderly population often have associated co-morbidities and poorer physical health, which would suggest that ERCP may be technically more challenging, or of higher risk in this group. We look at the safety of ERCP in patients over 90

Methods Patients aged 90 or above who underwent ERCP from October 1999 to October 2012 were studied retrospectively. The data was extracted from a single centre computer ERCP database.

Results ERCPs performed in patients aged 90 or above represented $6.5 \%$ of a total of 4017 procedures. The age range of patients was from 90 to 103 years. Therapeutic intervention was required in $246 / 262$ procedures (93.9\%). 16 specific types of intervention were performed, with the majority being sphincterotomy $(62.2 \%)$ or stent insertion $(16.7 \%)$. The procedure related mortality was $0.76 \%$ (2/262). 1 death occurred following a significant bleed post sphincterotomy, the other death resulted from a retroperitoneal perforation following duodenal dilatation. Complications occurred in $5.3 \%$ $(14 / 262)$ of procedures. The most frequently occurring complications were bleeding and cholangitis, each of which occurred in $3 / 262(1.1 \%)$ patients. Perforation occurred in $2 / 262$ patients $(0.76 \%)$. Pancreatitis did not occur in any of the patients. There were no complications related to co-morbidity.

Conclusion The number of ERCPs being performed in elderly patients is likely to increase. The procedure related mortality and morbidity is comparable with national figures. ERCP in this group is safe, practical and has a definite therapeutic benefit in a large proportion of patients.

Disclosure of Interest None Declared.

\section{PTH-045 ENDOSCOPIC SUBMUCOSAL DISSECTION FOR EARLY GASTRIC NEOPLASIA; PROSPECTIVE OUTCOMES FROM A SINGLE TERTIARY CENTRE}

doi:10.1136/gutjnl-2013-304907.532

1,2, $\mathrm{R} \mathrm{J}$ Haidry, ${ }^{3,4} \mathrm{M}$ Butt, ${ }^{4,5} \mathrm{~J}$ Louis-Auguste, ${ }^{6} \mathrm{M}$ Novelli, ${ }^{3,4} \mathrm{~L}$ Lovat, ${ }^{4} \mathrm{M}$ Banks. 'University College Hospital London (UCLH) NHS foundation Trust, Lonodn; ${ }^{2}$ National Medical laser Centre (NMLC), University College London; ${ }^{3} \mathrm{NMLC}$, London; ${ }^{4} \mathrm{UCLH}$, Iondon; ${ }^{5} \mathrm{NMLC}$, Lonodn; ${ }^{6}$ Department of Histopathology, UCLH, London, UK 